Relations industrielles

Industrial Relations

\title{
Valeurs économiques et valeurs juridiques dans les fusions d'entreprises, par Philippe Comte, Paris. Entreprise Moderne d'Édition, 4, rue Cambon, 1970, 269 pages.
}

\section{André Roy}

Volume 26, numéro 2, 1971

URI : https://id.erudit.org/iderudit/028238ar

DOI : https://doi.org/10.7202/028238ar

Aller au sommaire du numéro

Éditeur(s)

Département des relations industrielles de l'Université Laval

ISSN

0034-379X (imprimé)

1703-8138 (numérique)

Découvrir la revue

Citer ce compte rendu

Roy, A. (1971). Compte rendu de [Valeurs économiques et valeurs juridiques dans les fusions d'entreprises, par Philippe Comte, Paris. Entreprise Moderne d’Édition, 4, rue Cambon, 1970, 269 pages.] Relations industrielles / Industrial Relations, 26(2), 517-517. https://doi.org/10.7202/028238ar

Tous droits réservés @ Département des relations industrielles de l'Université Laval, 1971
Ce document est protégé par la loi sur le droit d'auteur. L'utilisation des services d'Érudit (y compris la reproduction) est assujettie à sa politique d'utilisation que vous pouvez consulter en ligne.

https://apropos.erudit.org/fr/usagers/politique-dutilisation/ 
expression nouvelle: la zone naturelle d'information (ZNI). En applicant ce principe à l'échelle de l'entreprise, on en arrive à la définition d'une autre expression : la base commune des données (BCD). Ces données communes à toute l'entreprise peuvent subir autant de traitements différents que l'exigent les services utilisateurs.

Les modalités de mise en cuvre du système intégré sont relativement faciles à saisir :

- Dans une première phase, on effectue un diagnostic de la nature de l'information habituellement utilisée dans la prise de décision et le contrôle à tous les niveaux.

- La deuxième phase consiste dans la détermination des zones naturelles d'information. C'est le regroupement des informations selon les fonctions.

- La mise sur pied d'un département d'informatique de même que l'inventaire de l'équipement nécessaire constituent la troisième phase.

- Vient ensuite la conception proprement dite d'un système intégré.

- Enfin, c'est la concrétisation du système et lancement de son exploitation.

C'est une étude qu'on peut lire avec profit, même si on n'est pas familier avec le language de l'ordinateur et la complexité des opérations qu'il peut effectuer.

Laurent BELANGER

Valeurs économiques et valeurs juridiques dans les fusions d'entreprises, par Philippe Comte, Paris. Entreprise Moderne d'Edition, 4, rue Cambon, 1970, 269 pages.

Depuis assez longtemps déjà, on assiste à un phénomène qui se généralise de plus en plus : la fusion ou le regroupement des entreprises. C'est ce phénomène qu'étudie Philippe Comte tant dans ses implications économiques que juridiques.

L'étude est divisée en trois parties: la première traite de ce que l'Auteur appelle « l'environnement économique des fusions d'entreprises 》; la deuxième est consacrée aux «valeurs économiques》 des fusions calculées en vue de la détermination des droits respectifs des actionnaires sur le nouvel ensemble; enfin, la troisième s'occupe des implications de nature juridique.

Comme il est facile de le deviner, les deux dernières parties de cet ouvrage ont un caractère technique marqué et s'adressent surtout aux spécialistes qui ont à analyser les obstacles qu'on peut rencontrer à l'occasion d'une fusion ainsi que les avantages qui peuvent en ressortir.

La première partie est d'une portée plus générale. L'auteur y étudie les causes générales des fusions et les obstacles qui s'y opposent, le rôle de la fusion d'entreprises parmi les modes de concentration à l'œuvre dans la société moderne, les conditions de réussite des fusions et l'attitude des pouvoirs publics.

André ROY

L'audiovisuel au service de la formation, méthodes-matériels, par Robinson $\mathbf{P}$. Rigg, Paris, Entreprise Moderne d'Edition, 1971, 220 pp.

Définitivement, les ouvrages de l'Entreprise moderne d'édition retiennent de plus en plus l'attention par leur qualité et la nature des sujets traités qui sont d'un grand intérêt pour ceux concernés par la gestion du personnel.

Le volume de R. P. Rigg originalement publié en anglais sous le titre Audiovisual Aids and Techniques in Managerial and Supervisory Training en 1969 est très bien traduit par José Ponzone.

Il faut dire dès maintenant que ce livre constitue presqu'une somme des techniques et des matériels de l'audiovisuel existant actuellement; ce qui se comprend assez bien étant donné d'une part, la nouveauté relative de cette aide pédagogique dans les procédés et méthodes de formation et, d'autre part, la compétence et l'expérience de l'auteur qui depuis 1945 a été mêlé à tout ce qui, de près ou de loin, concerne le mouvement audiovisuel dans les entreprises et les administrations en Grande-Bretagne.

L'auteur traite de six points principaux :

1. Le problème de la formation;

2. Du choix et de l'emploi des programmes de formation;

3. Les aides audiovisuelles et leurs utilisations ;

4. Techniques de présentation; 